V. Trivedi

Nagoya Math. J.

Vol. 185 (2007), 123-141

\title{
HILBERT-KUNZ MULTIPLICITY AND REDUCTION MOD $p$
}

\author{
V. TRIVEDI
}

\begin{abstract}
We show that the Hilbert-Kunz multiplicities of the reductions to positive characteristics of an irreducible projective curve in characteristic 0 have a well-defined limit as the characteristic tends to infinity.
\end{abstract}

Let $R$ be a Noetherian ring of prime characteristic $p>0$ and of dimension $d$ and let $I \subseteq R$ be an ideal of finite colength. Then we recall that the Hilbert-Kunz multiplicity of $R$ with respect to $I$ is defined as

$$
e_{H K}(R, I)=\lim _{n \rightarrow \infty} \frac{\ell\left(R / I^{\left[p^{n}\right]}\right)}{p^{n d}},
$$

where

$$
\begin{aligned}
I^{\left[p^{n}\right]} & =n \text {-th Frobenius power of } I \\
& =\text { ideal generated by } p^{n} \text {-th power of elements of } I
\end{aligned}
$$

is an ideal of finite colength and $\ell\left(R / I^{\left[p^{n}\right]}\right)$ denotes the length of the $R$ module $R / I^{\left[p^{n}\right]}$.

We note that this limit always exists as proved by Monsky. However, unlike Hilbert-Samuel multiplicity, this multiplicity could depend on the characteristic of the ring (see example of [HM] given here in Section 2).

In this paper, we study the behaviour of Hilbert-Kunz multiplicities (abbreviated henceforth to HK multiplicities) of the reductions to positive characteristics of an irreducible projective curve in characteristic 0 .

For instance, consider the following question. Let $f$ be a nonzero irreducible homogeneous element in the polynomial $\operatorname{ring} \mathbb{Z}\left[X_{1}, X_{2}, \ldots, X_{r}\right]$, and for any prime number $p \in \mathbb{Z}$, let $R_{p}=\mathbb{Z} / p \mathbb{Z}\left[X_{1}, X_{2}, \ldots, X_{r}\right] /(f)$ (this is the homogeneous coordinate ring of a projective variety over $\mathbb{Z} / p \mathbb{Z}$ ). Let

Received April 28, 2005.

Revised October 31, 2005, November 15, 2005.

2000 Mathematics Subject Classification: 13D40, 14H60, $13 \mathrm{H} 15$. 
$e_{H K}\left(R_{p}\right)$ denote the Hilbert-Kunz multiplicity of $R_{p}$ with respect to the graded maximal ideal. Then one can ask: does $\lim _{p \rightarrow \infty} e_{H K}\left(R_{p}\right)$ exist?

This question was first encountered by the author in a survey article [C], Problem 4, Section 5 (see also Remark 4.10 in [B1]). This seems a difficult question in general, as so far, there is no known general formula for HK multiplicity in terms of 'better understood' invariants. There does not seem to even be a heuristic argument as to why the limit should exist, in general, in arbitrary dimensions.

However in the case of a projective curve (equivalently 2 dimensional standard graded ring) over an algebraically closed field of characteristic $p>0$, one can express HK multiplicity in terms of (i) "standard" invariants of the curve which are constant in a flat family and (ii) normalized slopes of the quotients occuring in a strongly semistable Harder-Narasimhan filtration (HN filtration) (see Definitions 1.2 and 1.9) of the associated vector bundle on the curve (see [B1] and [T1]).

Hence, we may pose the question in the following more general setting. Given a projective curve $X$ defined over a field $k$ of char 0 with a vector bundle $V$ on $X$, let $\left(A, X_{A}, V_{A}\right)$ be a spread of the pair $(X, V)$ (details given above Proposition 2.2). For all closed points $s \in \operatorname{Spec} A$, let $V_{s}=V_{A} \otimes \overline{k(s)}$. Now for given $k \geq 0$ and each such $V_{s}$, let

$$
0 \subset F_{1}^{s} \subset \cdots \subset F_{t_{s}}^{s} \subset F_{t_{s}+1}^{s}=F^{k *} V_{s}
$$

be the HN filtration of $F^{k *} V_{s}$. Denote

$$
\begin{aligned}
& r_{i}\left(F^{k *} V_{s}\right)=\operatorname{rank}\left(\frac{F_{i}^{s}}{F_{i-1}^{s}}\right) \text { and } \\
& \text { the normalized slope } a_{i}\left(F^{k *} V_{s}\right)=\frac{1}{p^{k}} \mu\left(\frac{F_{i}^{s}}{F_{i-1}^{s}}\right) .
\end{aligned}
$$

Let $s_{0} \in \operatorname{Spec} A$ be the generic point of $\operatorname{Spec} A$. Then the question is:

$$
\text { For given } k \geq 0 \text {, does } \lim _{s \rightarrow s_{0}} \sum_{i} r_{i}\left(F^{k *} V_{s}\right) a_{i}\left(F^{k *} V_{s}\right)^{2} \text { exist? }
$$

We approach the question as follows. Following the notation of [L], for a vector bundle $V$ on a nonsingular projective curve $X$ in characteristic $p$, we attach convex polygons as follows. Consider the HN filtration

$$
0=E_{0} \subset E_{1} \subset \cdots \subset E_{l} \subset E_{l+1}=V
$$


of $V$. For $k \geq 0$, consider the HN filtration

$$
0=F_{0} \subset F_{1} \subset \cdots \subset F_{t} \subset F_{t+1}=F^{k *} V
$$

of the iterated Frobenius pull back bundle $F^{k *} V$. Let $P\left(F_{i}\right)=\left(\operatorname{rank} F_{i}\right.$, $\left.\operatorname{deg} F_{i} / p^{k}\right)$ in $\mathbb{R}^{2}$. Let $H N P_{p^{k}}(V)$ be the convex polygon in $\mathbb{R}^{2}$ obtained by connecting $P\left(F_{0}\right), \ldots, P\left(F_{t+1}\right)$ successively by line segments, and connecting the last one with the first one.

Let $p \geq 4(\operatorname{genus}(X)-1)(\operatorname{rank} V)^{3}$. Then we prove (Lemma 1.8) that the vertices of $H N P_{p^{k-1}}(V)$ are retained as a subset of the vertices of $H N P_{p^{k}}(V)$ and hence $H N P_{p^{k}}(V) \supset H N P_{p^{0}}(V)$. In particular, for $k \gg 0$, the HN filtration of the bundle $F^{k *}(V)$ is strongly semistable, therefore Theorem 2.7 of $[\mathrm{L}]$ comes as a corollary, in this case.

Now, for every vector bundle $F_{j}$ of the HN filtration of $F^{k *}(V)$, if we denote the slope of the line segment, joining $P\left(F_{j-1}\right)$ and $P\left(F_{j}\right)$, by $\mu_{j}\left(F^{k *}(V)\right) / p^{k}$ (see Notation 1.4), and if $E_{i}$ denotes the unique vector bundle occuring in the HN filtration of $V$ such that $F_{j}$ 'almost descends to' $E_{i}$ (see Definition 1.12), then we prove (Lemma 1.14) that

$$
\mu_{j}\left(F^{k *} V\right) / p^{k}=\mu_{i}(V)+O\left(\frac{1}{p}\right) .
$$

Hence $\lim _{p \rightarrow \infty}$ Area $H N P_{p^{k}}(V)=$ Area $H N P_{p^{0}}(V)$. In both Lemmas 1.8 and 1.14 we make crucial use of a result from the paper [SB] of ShepherdBarron.

Now, following the notation set up for the question (0.1), if we take a vector bundle $F_{j}^{s}$ occuring in the $\mathrm{HN}$ filtration of $F^{k *}\left(V_{s}\right)$ such that it almost descends to a vector bundle $E_{i}^{s}$ occuring in the HN filtration of $V_{s}$ then we get

$$
a_{j}\left(F^{k *}\left(V_{s}\right)\right):=\frac{\mu_{j}\left(F^{k *} V_{s}\right)}{p^{k}}=\mu_{i}\left(V_{s}\right)+O\left(\frac{1}{p}\right),
$$

where $p=\operatorname{char} k(s)$. From this we conclude (Proposition 2.2) that the question (0.1) has an affirmative answer.

In particular (Theorem 2.4) the Hilbert-Kunz multiplicities of the reductions to positive characteristics of an irreducible projective curve in characteristic 0 have a well-defined limit as the characteristic tends to $\infty$. This limit, which is (relatively) an easier invariant to compute, is a lower bound for the HK multiplicities of the reductions $(\bmod p)$, though examples of Han-Monsky show that the convergence is not monotonic as $p \rightarrow \infty$, in general (see Remark 2.7). 


\section{$\S 1$. The HN slope of $F^{*} V$ in terms of the HN slope of $V$}

Let $X$ be a nonsingular projective curve of genus $g \geq 1$, over an algebraically closed field $k$ of characteristic $p>0$. We recall the following definitions.

DeFinition 1.1. Let $V$ be a vector bundle (i.e., locally free coherent sheaf of $\mathcal{O}_{X}$-modules) on $X$. We say $V$ is a semistable vector bundle on $X$ if, for every subsheaf of $\mathcal{O}_{X}$-modules $F \subseteq V$, we have

$$
\mu(F):=\frac{\operatorname{deg} F}{\operatorname{rank} F} \leq \mu(V),
$$

where for a rank $r$ vector bundle $V$ on $X$ we define

$$
\operatorname{deg} V=\text { degree of the line bundle } \bigwedge^{r} V \text { on } X \text {. }
$$

Definition 1.2. Let $V$ be a vector bundle on $X$. A filtration of $V$ by vector subbundles

$$
0=E_{0} \subset E_{1} \subset \cdots \subset E_{l} \subset E_{l+1}=V
$$

is a Harder-Narasimhan filtration if

(1) the vector bundles $E_{1}, E_{2} / E_{1}, \ldots, E_{l+1} / E_{l}$ are all semistable.

(2) $\mu\left(E_{1}\right)>\mu\left(E_{2} / E_{1}\right)>\cdots>\mu\left(E_{l+1} / E_{l}\right)$.

Remark 1.3. For any Harder-Narasimhan filtration (we would call it HN filtration from now onwards), denoted as in Equation (1.1), the following is true (see [HN], Lemma 1.3.7),

(1) the filtration always exists and is unique for given $V$,

(2) $\mu\left(E_{1}\right)>\mu\left(E_{2}\right)>\cdots>\mu\left(E_{l+1}\right)=\mu(V)$,

(3) $\mu\left(E_{i} / E_{i-1}\right) \geq \mu(V) \geq \mu\left(E_{i+1} / E_{i}\right)$, for some $1 \leq i \leq l$.

NOTATION 1.4. If

$$
0=E_{0} \subset E_{1} \subset E_{2} \subset \cdots \subset E_{l} \subset E_{l+1}=V
$$

is the HN filtration for a vector bundle $V$ on $X$ then we denote

$$
\mu_{i}(V)=\mu\left(\frac{E_{i}}{E_{i-1}}\right), \quad \mu_{\max }(V)=\mu\left(E_{1}\right) \text { and } \mu_{\min }(V)=\mu\left(\frac{V}{E_{l}}\right) .
$$


Lemma 1.5. Let $V$ be a vector bundle over $X$ of rank $r$ and let

$$
0=E_{0} \subset E_{1} \subset E_{2} \subset \cdots \subset E_{l} \subset E_{l+1}=V
$$

be the $H N$ filtration of $V$. Then

$$
r^{3}>\frac{r-1}{\mu_{i}(V)-\mu_{i+1}(V)} .
$$

Proof. Let $\mu_{i}=\mu_{i}(V)$. Let us denote $\bar{r}_{i}=\operatorname{rank} E_{i} / E_{i-1}$ and $\bar{d}_{i}=$ $\operatorname{deg} E_{i} / E_{i-1}$. Then

$$
\frac{r-1}{\mu_{i}-\mu_{i+1}}=\frac{r-1}{\bar{d}_{i} / \bar{r}_{i}-\bar{d}_{i+1} / \bar{r}_{i+1}}=\frac{(r-1) \bar{r}_{i} \bar{r}_{i+1}}{\bar{d}_{i} \bar{r}_{i+1}-\bar{d}_{i+1} \bar{r}_{i}} .
$$

But

$$
\mu_{i}-\mu_{i+1}>0 \Longrightarrow \bar{d}_{i} \bar{r}_{i+1}-\bar{d}_{i+1} \bar{r}_{i}>0 \Longrightarrow \bar{d}_{i} \bar{r}_{i+1}-\bar{d}_{i+1} \bar{r}_{i} \geq 1 .
$$

Therefore

$$
\frac{r-1}{\mu_{i}-\mu_{i+1}} \leq(r-1) \bar{r}_{i} \bar{r}_{i+1}<r^{3} .
$$

This proves the lemma.

Definition 1.6. If $X$ is a projective variety defined over an algebraically closed field of characteristic $p>0$, then the absolute Frobenius morphism $F: X \rightarrow X$ is a morphism of schemes which is identity on the underlying set of $X$ and on the underlying sheaf of rings $F^{\#}: \mathcal{O}_{X} \rightarrow \mathcal{O}_{X}$ is the $p^{\text {th }}$ power map.

Remark 1.7. For a vector bundle $V$ on $X$, the Frobenius pull back $F^{*} V$ is a vector bundle on $X$ and

$$
\operatorname{rank} F^{*} V=\operatorname{rank} V \text { and } \mu\left(F^{*} V\right)=p \mu(V) .
$$

We recall the following crucial result by Shepherd-Barron.

COROLlaRY $2^{p}$. ([SB]) If $X$ is a nonsingular projective curve of genus $g$ and if $V$ is a semistable vector bundle on $X$ of rank $r$ such that $F^{*} V$ is not semistable then

$$
0<\mu_{\max }\left(F^{*} V\right)-\mu_{\min }\left(F^{*} V\right) \leq(2 g-2)(r-1) .
$$


Now we prove the following crucial lemma.

Lemma 1.8. Let $V$ be a vector bundle on $X$ with the $H N$ filtration as in Lemma 1.5. Assume that char $k=p>4(g-1) r^{3}$. Then,

$$
F^{*} E_{1} \subset F^{*} E_{2} \subset \cdots \subset F^{*} E_{l} \subset F^{*} V
$$

is a subfiltration of the $H N$ filtration of $F^{*} V$, that is, if

$$
0 \subset \widetilde{E}_{1} \subset \cdots \subset \widetilde{E}_{l_{1}+1}=F^{*} V
$$

is the $H N$ filtration of $F^{*} V$ then for every $1 \leq i \leq l$ there exists $1 \leq j_{i} \leq l_{1}$ such that $F^{*} E_{i}=\widetilde{E}_{j_{i}}$.

Proof. For each $0 \leq i \leq l$, let

$$
F^{*} E_{i} \subset E_{i 1} \subset \cdots \subset E_{i t_{i}} \subset F^{*} E_{i+1}
$$

be a filtration of vector bundles on $X$ such that

$$
0 \subset \frac{E_{i 1}}{F^{*} E_{i}} \subset \frac{E_{i 2}}{F^{*} E_{i}} \subset \cdots \subset \frac{F^{*} E_{i+1}}{F^{*} E_{i}}
$$

is the $\mathrm{HN}$ filtration of $F^{*}\left(E_{i+1} / E_{i}\right)$. Now it is enough to prove the

Claim.

$$
\begin{aligned}
0 \subset E_{01} \subset \cdots \subset E_{0 t_{0}} \subset F^{*} E_{1} \subset \cdots \subset & F^{*} E_{i} \subset E_{i 1} \subset \cdots \\
& \cdots \subset E_{i t_{i}} \subset F^{*} E_{i+1} \subset \cdots \subset F^{*} V
\end{aligned}
$$

is the $H N$ filtration of $F^{*} V$.

Proof of the claim. By construction, for $0 \leq i \leq l$ and for $1 \leq j<t_{i}$, we have

$$
\mu\left(\frac{E_{i j}}{E_{i, j-1}}\right)>\mu\left(\frac{E_{i, j+1}}{E_{i j}}\right)
$$

and

$$
\frac{E_{i j}}{E_{i, j-1}}, \frac{F^{*} E_{i}}{E_{i-1, t_{i-1}}} \text { and } \frac{E_{i 1}}{F^{*} E_{i}}
$$

are semistable. Hence, by Definition 1.2, it is enough to prove that

$$
\mu\left(\frac{F^{*} E_{i}}{E_{i-1, t_{i-1}}}\right)>\mu\left(\frac{E_{i 1}}{F^{*} E_{i}}\right) .
$$


Now, by Corollary $2^{p}$ of $[\mathrm{SB}]$, we have

$$
0 \leq \mu_{\max } F^{*}\left(\frac{E_{i+1}}{E_{i}}\right)-\mu_{\min } F^{*}\left(\frac{E_{i+1}}{E_{i}}\right) \leq(2 g-2)(r-1) .
$$

By Remark 1.3, for all $0 \leq i \leq l$, we have

$$
\mu_{\max } F^{*}\left(\frac{E_{i+1}}{E_{i}}\right) \geq \mu\left(F^{*}\left(\frac{E_{i+1}}{E_{i}}\right)\right) \geq \mu_{\min } F^{*}\left(\frac{E_{i+1}}{E_{i}}\right) .
$$

Therefore

$$
0 \leq \mu_{\max } F^{*}\left(\frac{E_{i+1}}{E_{i}}\right)-\mu\left(F^{*}\left(\frac{E_{i+1}}{E_{i}}\right)\right) \leq(2 g-2)(r-1) .
$$

Let $\mu_{i}=\mu_{i}(V)$. Then we have

$$
0 \leq \mu\left(\frac{E_{i 1}}{F^{*} E_{i}}\right)-p \mu_{i+1} \leq(2 g-2)(r-1) .
$$

Similarly

$$
0 \leq \mu\left(F^{*}\left(\frac{E_{i}}{E_{i-1}}\right)\right)-\mu_{\min }\left(F^{*}\left(\frac{E_{i}}{E_{i-1}}\right)\right) \leq(2 g-2)(r-1)
$$

which means

$$
0 \leq p \mu_{i}-\mu\left(\frac{F^{*} E_{i}}{E_{i-1, t_{i-1}}}\right) \leq(2 g-2)(r-1) .
$$

Now, multiplying (1.3) and (1.4) by -1 and adding, we get

$$
\begin{aligned}
-4(g-1)(r-1)+p\left(\mu_{i}-\mu_{i+1}\right) & \leq \mu\left(\frac{F^{*} E_{i}}{E_{i-1, t_{i-1}}}\right)-\mu\left(\frac{E_{i 1}}{F^{*} E_{i}}\right) \\
& \leq p\left(\mu_{i}-\mu_{i+1}\right) .
\end{aligned}
$$

Since $p>4(g-1) r^{3}$, Lemma 1.5 implies that

$$
-4(g-1)(r-1)+p\left(\mu_{i}-\mu_{i+1}\right)>0,
$$

and hence

$$
\mu\left(\frac{F^{*} E_{i}}{E_{i-1, t_{i-1}}}\right)>\mu\left(\frac{E_{i 1}}{F^{*} E_{i}}\right),
$$

This proves the claim, and hence the lemma. 
Definition 1.9. (1) A vector bundle $V$ on $X$ is strongly semistable if $F^{s *}(V)$ is semistable for every $s^{\text {th }}$ iterated power of the absolute Frobenius map $F: X \rightarrow X$.

(2) A filtration by subbundles

$$
0=E_{0} \subset E_{1} \subset \cdots \subset E_{l} \subset E_{l+1}=V
$$

of $V$ is a strongly semistable $H N$ filtration if

(a) it is the HN filtration and

(b) $E_{1}, E_{2} / E_{1}, \ldots, E_{l+1} / E_{l}$ are strongly semistable vector bundles.

Remark 1.10. (1) If the HN filtration

$$
0=E_{0} \subset E_{1} \subset \cdots \subset E_{l} \subset E_{l+1}=V
$$

of $V$ is strongly semistable then, for any $k \geq 0$, the filtration

$$
0=E_{0} \subset F^{k *} E_{1} \subset \cdots \subset F^{k *} E_{l} \subset F^{k *} E_{l+1}=F^{k *} V
$$

is the strongly semistable HN filtration of $F^{k *} V$.

(2) If $V$ is a rank 2 vector bundle on $X$ and is not semistable then its HN filtration will be strongly semistable; as it would be filtered by line bundles, which are always semistable and hence strongly semistable.

Remark 1.11. Note that, if $\operatorname{rank} V=r$ and char $k=p>4(g-1) r^{3}$, then Lemma 1.8 implies that there exists $s \geq 0$ such that the HN filtration of $F^{{ }^{*}} V$ is strongly semistable. Therefore, Theorem 2.7 of [L] follows in this case.

Definition 1.12. Let $E$ be a vector bundle on $X$. A vector bundle $F_{j} \neq 0$ occuring in the HN filtration of $F^{s *} E$ is said to almost descend to a bundle $E_{i}$ occuring in the HN filtration of $E$ if $F_{j} \subseteq F^{s *} E_{i}$ and $E_{i}$ is the smallest bundle in the HN filtration of $E$, with this property.

Remark 1.13. Note that, if $p>4(g-1)(\operatorname{rank} E)^{3}$, then by Lemma 1.8, we have the following transitivity property: if $F_{j}$ almost descends to a bundle $\widetilde{E}_{i}$ in the HN filtration of $F^{k *} E$, and $\widetilde{E}_{i}$ almost descends to a bundle $E_{t}$ occuring in the HN filtration of $E$, then $F_{j}$ almost descends to the bundle $E_{t}$. 
Lemma 1.14. Let $E$ be a vector bundle on $X$ of rank $r$ and let the characteristic $p$ satisfy $p>4(g-1) r^{3}$. Let $F_{j} \neq 0$ be a subbundle in the $H N$ filtration of $F^{s *} E$, which almost descends to a vector bundle $E_{i}$ occuring in the $H N$ filtration of $E$. Then

$$
\frac{\mu_{j}\left(F^{s *} E\right)}{p^{s}}=\mu_{i}(E)+\frac{C}{p}
$$

where $|C| \leq 4(g-1)(r-1)$, and $\mu_{j}\left(F^{s *} E\right)$ and $\mu_{i}(E)$ are given as in Notation 1.4.

Proof. Let $F_{j-1}$ be the vector bundle on $X$ such that $F_{j-1} \subset F_{j}$ are two consecutive subbundles of the HN filtration of $F^{s *} E$. Therefore, by Lemma 1.8, there exist two consecutive subbundles $E_{i_{1}-1} \subset E_{i_{1}}$ in the HN filtration of $F^{(s-1) *} E$ such that

$$
F^{*} E_{i_{1}-1} \subseteq F_{j-1} \subset F_{j} \subseteq F^{*} E_{i_{1}} .
$$

In particular, we are in the situation that $E_{i_{1}} / E_{i_{1}-1}$ is a semistable vector bundle on $X$ and

(1) either $F_{j-1} / F^{*} E_{i_{1}-1}=0$ in $F^{*}\left(E_{i_{1}} / E_{i_{1}-1}\right)$, and $F_{j} / F^{*} E_{i_{1}-1}$ is the first nonzero vector bundle in the HN filtration of $F^{*}\left(E_{i_{1}} / E_{i_{1}-1}\right)$ or

(2) $F_{j-1} / F^{*} E_{i_{1}-1} \subset F_{j} / F^{*} E_{i_{1}-1}$ are two consecutive subbundles in the HN filtration of $F^{*}\left(E_{i_{1}} / E_{i_{1}-1}\right)$.

In both the cases, by Definition 1.2, we have

$$
\mu_{\min } F^{*}\left(\frac{E_{i_{1}}}{E_{i_{1}-1}}\right) \leq \mu\left(\frac{F_{j}}{F_{j-1}}\right) \leq \mu_{\max } F^{*}\left(\frac{E_{i_{1}}}{E_{i_{1}-1}}\right) .
$$

Therefore, Corollary $2^{p}$ of $[\mathrm{SB}]$ implies

$$
-2(g-1)(r-1) \leq \mu_{j}\left(F^{* *}(V)\right)-\mu\left(F^{*}\left(\frac{E_{i_{1}}}{E_{i_{1}}-1}\right)\right) \leq 2(g-1)(r-1) .
$$

Note that $\mu\left(F^{*}\left(E_{i_{1}} / E_{i_{1}-1}\right)\right)=p \mu_{i_{1}}\left(F^{(s-1) *} E\right)$. Therefore we have

$$
\mu_{j}\left(F^{s *} E\right)=p \mu_{i_{1}}\left(F^{(s-1) *} E\right)+C_{1},
$$

where $\left|C_{1}\right| \leq 2(g-1)(r-1)$. 
Note $E_{i_{1}}$ is a nonzero subbundle in the HN filtration of $F^{(s-1) *} E$ which almost descends to $E_{i}$ occuring in the HN filtration of $E$. Hence, inductively one can prove that

$$
\mu_{i_{1}}\left(F^{(s-1) *} E\right)=p^{s-1} \mu_{i}(E)+p^{s-2} C_{s}+\cdots+C_{2},
$$

where $\left|C_{2}\right|, \ldots,\left|C_{s}\right| \leq 2(g-1)(r-1)$. Therefore

$$
\mu_{j}\left(F^{s *} E\right)=p^{s} \mu_{i}(E)+p^{s-1} C_{s}+\cdots+p C_{2}+C_{1} .
$$

Therefore

$$
\frac{\mu_{j}\left(F^{s *} E\right)}{p^{s}}=\mu_{i}(E)+\frac{1}{p^{s}}\left(p^{s-1} C_{s}+\cdots+p C_{2}+C_{1}\right) .
$$

But

$$
\left|\left(p^{s-1} C_{s}+\cdots+p C_{2}+C_{1}\right)\right| \leq\left(1+\cdots+p^{s-1}\right)(2(g-1)(r-1)) .
$$

Since $\left(1+p+\cdots+p^{s-1}\right) / p^{s-1} \leq 2$, we have

$$
\frac{\left|p^{s-1} C_{s}+\cdots+p C_{2}+C_{1}\right|}{p^{s-1}} \leq 4(g-1)(r-1) .
$$

Therefore we conclude that

$$
\frac{\mu_{j}\left(F^{s *} E\right)}{p^{s}}=\mu_{i}(E)+\frac{C}{p},
$$

where $|C| \leq 4(g-1)(r-1)$. This proves the lemma.

Notation 1.15. Henceforth we assume that the characteristic $p$ satisfies $p>4(g-1) r^{3}$. We also fix a vector bundle $V$ on $X$ of rank $r$ with the HN filtration

$$
0=E_{0} \subset E_{1} \subset E_{2} \subset \cdots \subset E_{l} \subset E_{l+1}=V .
$$

Let

$$
0 \subset F_{1} \subset F_{2} \subset \cdots \subset F_{t} \subset F_{t+1}=F^{k *} V
$$

be the HN filtration of $F^{k *} V$, and let

$$
r_{i}\left(F^{k *} V\right)=\operatorname{rank}\left(\frac{F_{i}}{F_{i-1}}\right) \text { and } a_{i}\left(F^{k *} V\right)=\frac{\mu_{i}\left(F^{k *} V\right)}{p^{k}} .
$$


Proposition 1.16. With the notation as above, where $p>4(g-1) r^{3}$, if a vector bundle $F_{j}$ of the $H N$ filtration of $F^{k *} V$ almost descends to a vector bundle $E_{i}$ of the $H N$ filtration of $V$ then, for any $m \geq 1$,

$$
a_{j}\left(F^{k *} V\right)^{m}=\mu_{i}(V)^{m}+\frac{C}{p}
$$

where $|C| \leq 8 g r\left(\max \left\{2\left|\mu_{1}(V)\right|, \ldots, 2\left|\mu_{l+1}(V)\right|, 2\right\}^{m-1}\right)$.

Proof. By Lemma 1.14, we have

$$
a_{j}\left(F^{k *} V\right)=\mu_{i}(V)+\frac{c_{i j}}{p}
$$

where $\left|c_{i j}\right| \leq 4(g-1)(r-1)$. For the sake of abbreviation let us denote $\mu_{j}(V)$ by $\mu_{j}$. Therefore

$$
a_{j}\left(F^{k *} V\right)^{m}-\mu_{i}^{m}=\left(\begin{array}{c}
m \\
1
\end{array}\right) \mu_{i}^{m-1} \frac{c_{i j}}{p}+\cdots+\left(\begin{array}{c}
m \\
m-1
\end{array}\right) \mu_{i} \frac{c_{i j}^{m-1}}{p^{m-1}}+\left(\begin{array}{c}
m \\
m
\end{array}\right) \frac{c_{i j}^{m}}{p^{m}} .
$$

Hence

$$
\begin{aligned}
& \left|a_{j}\left(F^{k *} V\right)^{m}-\mu_{i}^{m}\right| \\
& \quad \leq \frac{\left|c_{i j}\right|}{p}\left[\left(\begin{array}{c}
m \\
1
\end{array}\right)\left|\mu_{i}\right|^{m-1}+\cdots+\left(\begin{array}{c}
m \\
m-1
\end{array}\right)\left|\mu_{i}\right| \frac{\left|c_{i j}\right|^{m-2}}{p^{m-2}}+\left(\begin{array}{c}
m \\
m
\end{array}\right) \frac{\left|c_{i j}\right|^{m-1}}{p^{m-1}}\right] .
\end{aligned}
$$

Now, as $\left|c_{i j}\right| / p \leq 1$, this implies

$$
\left|a_{j}\left(F^{k *} V\right)^{m}-\mu_{i}^{m}\right| \leq \frac{\left|c_{i j}\right|}{p}\left[\left(\begin{array}{c}
m \\
1
\end{array}\right)\left|\mu_{i}\right|^{m-1}+\cdots+\left(\begin{array}{c}
m \\
m-1
\end{array}\right)\left|\mu_{i}\right|+\left(\begin{array}{c}
m \\
m
\end{array}\right)\right] .
$$

(1) Let $\left|\mu_{i}\right| \leq 1$. Then

$$
\begin{aligned}
\left|a_{j}\left(F^{k *} V\right)^{m}-\mu_{i}^{m}\right| & \leq \frac{\left|c_{i j}\right|}{p}\left[\left(\begin{array}{c}
m \\
1
\end{array}\right)+\cdots+\left(\begin{array}{c}
m \\
m-1
\end{array}\right)+\left(\begin{array}{c}
m \\
m
\end{array}\right)\right] \\
& \leq \frac{\left|c_{i j}\right|}{p}\left(2^{m}-1\right) \leq \frac{1}{p}\left(8 g r\left(2^{m-1}\right)\right) .
\end{aligned}
$$

(2) Let $\left|\mu_{i}\right| \geq 1$. Then

$$
\begin{aligned}
\left|a_{j}\left(F^{k *} V\right)^{m}-\mu_{i}^{m}\right| & \leq \frac{\left|c_{i j}\right|\left|\mu_{i}\right|^{m-1}}{p}\left[\left(\begin{array}{c}
m \\
1
\end{array}\right)+\cdots+\left(\begin{array}{c}
m \\
m-1
\end{array}\right)+\left(\begin{array}{c}
m \\
m
\end{array}\right)\right] \\
& \leq \frac{\left|c_{i j}\right|\left|\mu_{i}\right|^{m-1}}{p}\left(2^{m}-1\right) \leq \frac{1}{p}\left(8 g r\left(2\left|\mu_{i}\right|\right)^{m-1}\right) .
\end{aligned}
$$

Hence the proposition. 


\section{§2. Applications}

We extend Notation 1.15 to the case, when the underlying field is of arbitrary characteristic, as follows.

Notation 2.1. Let $X$ be a nonsingular curve over an algebraically closed field $k$ and $V$ a vector bundle on $X$, with HN filtration

$$
0=E_{0} \subset E_{1} \subset \cdots \subset E_{l} \subset E_{l+1}=V .
$$

(1) If char $k=p>0$, then we define the numbers $\mu_{i}\left(F^{k *} V\right), r_{i}\left(F^{k *} V\right)$ and $a_{i}\left(F^{k *} V\right)$ as in Notations 1.4 and 1.15. Moreover, we choose an integer $s \geq 0$ such that $F^{s *}(V)$ has a strongly semistable HN filtration and we denote

$$
\tilde{a}_{i}(V)=a_{i}\left(F^{s *}(V)\right) \text { and } \widetilde{r}_{i}(V)=r_{i}\left(F^{s *}(V)\right)
$$

(note that, by Remark 1.10, these numbers are independent of the choice of such an $s$ ).

(2) If char $k=0$, define

$$
\widetilde{a}_{i}(V)=\mu_{i}(V)=\mu\left(\frac{E_{i}}{E_{i-1}}\right), \text { and } \widetilde{r}_{i}(V)=r_{i}(V)=\operatorname{rank}\left(\frac{E_{i}}{E_{i-1}}\right) .
$$

Here we recall a notion of spread for the pair $(X, V)$, where $X$ is a nonsingular curve over a field of characteristic 0 and $V$ is a vector bundle on $X$. For such a pair there exists a finitely generated $\mathbb{Z}$-algebra $A \subseteq k$ and a projective $A$-scheme $X_{A}$ over $A$ and coherent, locally free sheaves $V_{A}$ and

$$
E_{1 A} \subset \cdots \subset E_{l A} \subset V_{A}
$$

on $X_{A}$ such that

$$
X_{A} \times_{\operatorname{Spec} A} \operatorname{Spec} k=X \text { and } V_{A} \otimes_{A} k=V,
$$

and for all closed points $s \in \operatorname{Spec} A$, if

$$
V_{s}=V_{A} \otimes \overline{k(s)}, \text { and } E_{i(s)}=E_{i A} \otimes \overline{k(s)},
$$

then

$$
0 \subset E_{1(s)} \subset \cdots \subset E_{l(s)} \subset V_{s}
$$


is the HN filtration of $V_{s}$ (this follows by an openness property of semistable vector bundles $([\mathrm{Ma}]))$. We call the triple $\left(A, X_{A}, V_{A}\right)$ a spread of $(X, V)$.

Moreover, if, for the pair $(X, V)$, we have a spread $\left(A, X_{A}, V_{A}\right)$ as above and $A \subset A^{\prime} \subset k$, for some finitely generated $\mathbb{Z}$-algebra $A^{\prime}$ then $\left(A^{\prime}, X_{A^{\prime}}, V_{A^{\prime}}\right)$ satisfy the same properties as $\left(A, X_{A}, V_{A}\right)$. Hence we may always assume that the spread $\left(A, X_{A}, V_{A}\right)$ as above is chosen such that $A$ contains a given finitely generated algebra $A_{0} \subseteq k$.

Proposition 2.2. Let $f: X_{A} \rightarrow \operatorname{Spec} A$ be a projective morphism of Noetherian schemes, smooth of relative dimension 1, where $A$ is a finitely generated $\mathbb{Z}$-algebra and is an integral domain. Let $\mathcal{O}_{X_{A}}(1)$ be an $f$-very ample invertible sheaf on $X_{A}$. Let $V_{A}$ be a vector bundle on $X_{A}$. For $s \in \operatorname{Spec} A$, let $V_{s}=V_{A} \otimes_{A} \overline{k(s)}$ be the induced vector bundle on the smooth projective curve $X_{s}=X_{A} \otimes_{A} \overline{k(s)}$. Let $s_{0}=\operatorname{Spec} Q(A)$ be the generic point of $\operatorname{Spec} A$. Then,

(1) for any $k \geq 0$ and $m \geq 0$, we have

$$
\lim _{s \rightarrow s_{0}} \sum_{j} r_{j}\left(F^{k *} V_{s}\right) a_{j}\left(F^{k *} V_{s}\right)^{m}=\sum_{i} r_{i}\left(V_{s_{0}}\right) \mu_{i}\left(V_{s_{0}}\right)^{m} .
$$

(2) Similarly

$$
\lim _{s \rightarrow s_{0}} \sum_{j} \widetilde{r}_{j}\left(V_{s}\right) \widetilde{a}_{j}\left(V_{s}\right)^{m}=\sum_{i} r_{i}\left(V_{s_{0}}\right) \mu_{i}\left(V_{s_{0}}\right)^{m},
$$

where in both the limits, s runs over closed points of $\operatorname{Spec} A$.

Proof. To prove the proposition, one can replace $\operatorname{Spec} A$ by an affine open subset (after localizing $A$ if necessary), so that

$$
\left(A, X_{A}, V_{A}\right) \text { is a spread of }\left(X_{A} \otimes k\left(s_{0}\right), V_{A} \otimes k\left(s_{0}\right)\right)
$$

as defined above. Moreover we can choose $A$ such that, for any closed point $s \in \operatorname{Spec} A$, we have

$$
\operatorname{char} k(s)>4\left(\text { genus } X_{s}-1\right)\left(\operatorname{rank} V_{s}\right)^{3}=4\left(\text { genus } X_{s_{0}}-1\right)\left(\operatorname{rank} V_{s_{0}}\right)^{3} .
$$

Therefore, if we denote

$$
M=8\left(\operatorname{genus}\left(X_{s_{0}}\right)\right) r\left(V_{s_{0}}\right)\left(\max \left\{2,2\left|\mu_{1}\left(V_{s_{0}}\right)\right|, \ldots,\left|\mu_{l+1}\left(V_{s_{0}}\right)\right|\right\}^{m-1}\right),
$$


where $r\left(V_{s_{0}}\right)=\operatorname{rank}\left(V_{s_{0}}\right)$, then, by Proposition 1.16, we have

$$
\begin{aligned}
\sum_{j} r_{j}\left(F^{k *} V_{s}\right) a_{j}\left(F^{k *} V_{s}\right)^{m} & =\sum_{i} r_{i}\left(V_{s}\right)\left(\mu_{i}\left(V_{s}\right)^{m}+\frac{C_{i}}{p}\right), \text { where }\left|C_{i}\right| \leq M \\
& =\sum_{i} r_{i}\left(V_{s_{0}}\right) \mu_{i}\left(V_{s_{0}}\right)^{m}+\frac{C_{s_{k}}}{p}
\end{aligned}
$$

where $\left|C_{s_{k}}\right| \leq r\left(V_{s_{0}}\right) M$. In particular, for every closed point $s \in \operatorname{Spec} A$, we have

$$
\sum_{j} \widetilde{r}_{j}\left(V_{s}\right) \widetilde{a}_{j}\left(V_{s}\right)^{m}=\sum_{i} r_{i}\left(V_{s_{0}}\right) \mu_{i}\left(V_{s_{0}}\right)^{m}+\frac{C_{s}}{p},
$$

where $\left|C_{s}\right| \leq r\left(V_{s_{0}}\right) M$. Now the proposition follows easily.

Corollary 2.3. Along with Notation 2.1, if we denote (as defined in [B2]), for char $k>0, \mu_{H K}(V)=\sum_{i} \widetilde{r}_{i}(V) \widetilde{a}_{i}(V)^{2}$, and for char $k=0$, $\mu_{H K}(V)=\sum_{j} r_{j}(V) \mu_{j}(V)^{2}$, then

$$
\lim _{s \rightarrow s_{0}} \mu_{H K}\left(V_{s}\right)=\mu_{H K}\left(V_{s_{0}}\right) .
$$

Proof. The corollary follows by substituting $m=2$ in the second statement of Proposition 2.2.

Here recall similar notion of spread for the pair $(R, I)$, where $R$ is a finitely generated $\mathbb{N}$-graded two dimensional domain over an algebraically closed field $k$ of characteristic 0 and $I \subset R$ is a homogeneous ideal of finite colength. For such a pair, there exists a finitely generated $\mathbb{Z}$-algebra $A \subseteq k$, a finitely generated $\mathbb{N}$-graded algebra $R_{A}$ over $A$ and a homogeneous ideal $I_{A} \subset R_{A}$ such that $R_{A} \otimes_{A} k=R$ and for any closed point $s \in \operatorname{Spec} A$ (i.e. maximal ideal of $A$ ) the ring $R_{s}=R_{A} \otimes_{A} k(s)$ is a finitely generated $\mathbb{N}$-graded 2-dimensional domain (which is a normal domain if $R$ is normal) over $k(s)$ and the ideal $I_{s}=\operatorname{Im}\left(I_{A} \otimes_{A} k(s)\right) \subset R_{s}$ is a homogeneous ideal of finite colength. We call $\left(A, R_{A}, I_{A}\right)$ a spread of the pair $(R, I)$.

Moreover, if, for the pair $(R, I)$, we have a spread $\left(A, R_{A}, I_{A}\right)$ as above and $A \subset A^{\prime} \subset k$, for some finitely generated $\mathbb{Z}$-algebra $A^{\prime}$ then $\left(A^{\prime}, R_{A^{\prime}}, I_{A^{\prime}}\right)$ satisfy the same properties as $\left(A, R_{A}, I_{A}\right)$. Hence we may always assume that the spread $\left(A, R_{A}, I_{A}\right)$ as above is chosen such that $A$ contains a given finitely generated algebra $A_{0} \subseteq k$. 
TheOrem 2.4. Let $R$ be a standard graded two dimensional domain over an algebraically closed field $k$ of characteristic 0 . Let $I \subset R$ be a homogeneous ideal of finite colength. Let $\left(A, R_{A}, I_{A}\right)$ be a spread as given above. Then

$$
\lim _{s \rightarrow s_{0}} e_{H K}\left(R_{s}, I_{s}\right)
$$

exists and is a rational number, where $s_{0}=\operatorname{Spec} Q(A)$ is the generic point of $\operatorname{Spec} A$, and the limit is taken over closed points $s \in \operatorname{Spec} A$.

Proof. Let $R \rightarrow S$ be the normalization of $R$. Then $R \rightarrow S$ is a finite graded map of degree 0 , and $Q(R)=Q(S)$, such that $S$ is a finitely generated $\mathbb{N}$-graded 2-dimensional normal domain over $k$. Now, for pairs $(R, I),(S, I S)$, we choose spreads $\left(A, R_{A}, I_{A}\right)$ and $\left(A, S_{A}, I S_{A}\right)$ such that for every closed point $s \in \operatorname{Spec} A$, the natural map $R_{s}=R_{A} \otimes k(s) \rightarrow S_{s}=$ $S_{A} \otimes k(s)$ is a finite graded map of degree 0 . Therefore we have the following commutative diagrams of horizontal finite maps

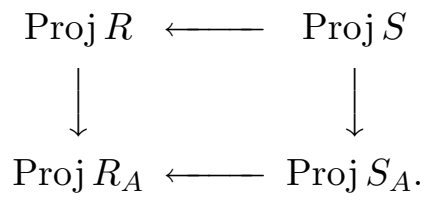

It follows that, for every $s \in \operatorname{Spec} A$, the corresponding map of curves

$$
\operatorname{Proj} S_{A} \otimes_{A} k(s) \longrightarrow \operatorname{Proj} R_{A} \otimes_{A} k(s)
$$

is a finite map, where the curve $\operatorname{Proj} S_{A} \otimes_{A} k(s)$ is nonsingular. Since $R_{s} \rightarrow S_{s}$ is a finite map such that $S_{s}$ is a module of rank 1 over $R_{s}$, by Lemma 1.3 in $[\mathrm{M}]$, Theorem 2.7 in [WY] and [BCP], we have

$$
e_{H K}\left(R_{s}, I_{s}\right)=e_{H K}\left(S_{s}, I S_{s}\right) \text {, for every closed point } s \in \operatorname{Spec} A \text {. }
$$

Therefore it is enough to prove the following

CLAIM. $\lim _{s \rightarrow s_{0}} e_{H K}\left(S_{s}, I S_{s}\right)$ exists.

Proof of the claim. Let $I$ and $I S_{A}$ be generated by the set $\left\{f_{1}, \ldots, f_{k}\right\}$, where $\operatorname{deg} f_{i}=d_{i}$. We have a short exact sequence of $\mathcal{O}_{X_{A}}$-sheaves (see [B1] and $[\mathrm{T} 1])$ :

$$
0 \longrightarrow V_{A} \longrightarrow \bigoplus_{i=1}^{k} \mathcal{O}_{X_{A}}\left(1-d_{i}\right) \longrightarrow \mathcal{O}_{X_{A}}(1) \longrightarrow 0
$$


where $\mathcal{O}_{X_{A}}\left(1-d_{i}\right) \rightarrow \mathcal{O}_{X_{A}}(1)$ is multiplication by $f_{i}$. Restricting (2.1) to the fiber $X_{s}$, we get

$$
0 \longrightarrow V_{s} \longrightarrow \bigoplus_{i=1}^{k} \mathcal{O}_{X_{s}}\left(1-d_{i}\right) \longrightarrow \mathcal{O}_{X_{s}}(1) \longrightarrow 0 .
$$

Note that (see [B1] and [T1]),

$$
e_{H K}\left(S_{s}, I S_{s}\right)=\frac{\operatorname{deg} \operatorname{Proj} S_{s}}{2}\left(\sum_{i} \widetilde{r}_{i}\left(V_{s}\right) \widetilde{a}_{i}\left(V_{s}\right)^{2}-\sum_{i=1}^{k} d_{i}^{2}\right) .
$$

Therefore

$$
\lim _{s \rightarrow s_{0}} e_{H K}\left(S_{s}, I S_{s}\right)=\frac{\operatorname{deg} \operatorname{Proj} S}{2}\left(\lim _{s \rightarrow s_{0}} \sum_{i} \widetilde{r}_{i}\left(V_{s}\right) \widetilde{a}_{i}\left(V_{s}\right)^{2}-\sum_{i=1}^{k} d_{i}^{2}\right) .
$$

Hence, by Proposition 2.2,

$$
\lim _{s \rightarrow s_{0}} e_{H K}\left(S_{s}, I S_{s}\right)=\frac{\operatorname{deg} \operatorname{Proj} S}{2}\left(\sum_{i} r_{i}\left(V_{s_{0}}\right) \mu_{i}\left(V_{s_{0}}\right)^{2}-\sum_{i=1}^{k} d_{i}^{2}\right) .
$$

In particular $\lim _{s \rightarrow s_{0}} e_{H K}\left(S_{s}, I S_{s}\right)$ exists and is a rational number. This proves the theorem.

Remark 2.5. Let $R$ be a standard graded 2 dimensional domain over a field of characteristic 0 . Let $I \subset R$ be a homogeneous ideal of finite colength. Then for the pair $(R, I)$ we choose a spread $\left(A, X_{A}, I_{A}\right)$ as described earlier and define

$$
e_{H K}(R, I)=\lim _{s \rightarrow s_{o}} e_{H K}\left(R_{s}, I_{s}\right) .
$$

This is, inherently, a well defined notion (i.e., irrespective of a choice of generators of $I)$, since in positive characteristic $e_{H K}\left(R_{s}, I_{s}\right)$ is independent of a choice of generators of $I_{s}$. We extend this definition to a standard graded 2-dimensional ring $R$, over a field $k$ of characteristic 0 , and a homogeneous ideal $I \subset R$ of finite colength as

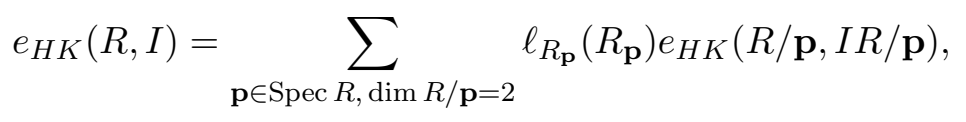


This is a always a rational number, by Theorem 2.4 .

Note that a notion of $e_{H K}(R, I)$, when $R$ is also a normal domain (i.e., $\operatorname{Proj} R$ is a smooth curve) over a field of characteristic 0 , is given in [B2] as

$$
e_{H K}(R, I)=\frac{\operatorname{deg} \operatorname{Proj} R}{2}\left(\mu_{H K}(V)-\sum_{i=1}^{k} d_{i}^{2}\right),
$$

where $V$ is the vector bundle given by

$$
0 \longrightarrow V \longrightarrow \bigoplus_{i} \mathcal{O}_{X}\left(1-d_{i}\right) \longrightarrow \mathcal{O}_{X}(1) \longrightarrow 0
$$

By Corollary 2.3, these two definitions (2.3) and (2.2) coincide, in this case.

Remark 2.6. It follows from Remark 4.13 of [T1] that, for every closed point $s$ in $\operatorname{Spec} A$, where $\left(A, R_{A}, I_{A}\right)$ is a spread for the pair $(R, I)$, we have

$$
e_{H K}\left(R_{s}, I_{s}\right) \geq e_{H K}(R, I),
$$

and $e_{H K}\left(R_{s}, I_{s}\right)=e_{H K}(R, I)$ if and only if $\mathrm{HN}$ filtration of $V_{s}$ is the strongly semistable HN filtration, where $e_{H K}\left(R_{s}, I_{s}\right)$ is the HK multiplicity defined (as given in the introduction) over the residue field $k(s)$, of the point $s$, which is of positive characteristic and $e_{H K}(R, I)$ is defined (as in Remark 2.5), over the quotient field of $A$ which is of characteristic 0 . If $V_{s}$ is semistable then

$$
e_{H K}(R, I)=\frac{\operatorname{deg} \operatorname{Proj} R_{s}}{2}\left(\left(\sum_{i} d_{i}\right)^{2} /(t-1)-\sum_{i} d_{i}^{2}\right) .
$$

Remark 2.7. As observed in the above remark,

$$
\left\{e_{H K}\left(R_{s}, I_{s}\right)-e_{H K}(R, I) \mid s \in\{\text { closed points of } \operatorname{Spec} A\}\right\}
$$

is a sequence of non-negative rational numbers (indexed by the closed points of Spec $A$ ), converging to 0. Examples show that it could be oscillating.

First we recall the following result of [T2]

Corollary. Let $X_{p}=\operatorname{Proj} R_{p}$, where $\left.R_{p}=k[x, y, z] /(f)\right)$, be a nonsingular plane curve of degree $d$ over an algebraically closed field $k$ of characteristic $p>0$. Then

$$
e_{H K}\left(X_{p}, \mathcal{O}_{X_{p}}(1)\right)=e_{H K}\left(R_{p},(x, y, z) R_{p}\right)=\frac{3 d}{4}+\frac{l^{2}}{4 d p^{2 s}},
$$


where $s \geq 1$ is a number such that $F^{(s-1) *} V_{X_{p}}$ is semistable and $F^{s *} V_{X_{p}}$ is not semistable (if $F^{t *} V_{X_{p}}$ is semistable for all $t \geq 0$, we take $s=\infty$ ) and $l$ is an integer congruent to $p d(\bmod 2)$ with $0 \leq l \leq d(d-3)$.

Monsky (around 1990) calculated the Hilbert Kunz function for plane curves $k[x, y, z] /\left(x^{d}+y^{d}+z^{d}\right)$, this result was later generalized in $[\mathrm{H}]$ and $[\mathrm{HM}]$ to diagonal hypersurfaces $k\left[x_{1}, x_{2}, \ldots\right] /\left(\sum_{i} x_{i}^{d}\right)$. In particular, arguing as in the examples of $[\mathrm{HM}]$ we have the following

Let

$$
R_{p}=k[X, Y, Z] /\left(x^{4}+y^{4}+z^{4}\right), \quad \text { where char } k=p .
$$

Then

$$
\begin{aligned}
e_{H K}\left(R_{p},(x, y, z) R_{p}\right) & =3+\frac{1}{p^{2}}, & & \text { if } p \equiv \pm 3(8) \\
& =3, & & \text { if } p \equiv \pm 1(8) .
\end{aligned}
$$

Now, let $X_{p}=\operatorname{Proj} R_{p}$. Consider the short exact sequence

$$
0 \longrightarrow V_{X_{p}} \longrightarrow \mathcal{O}_{X_{p}} \oplus \mathcal{O}_{X_{p}} \oplus \mathcal{O}_{X_{p}} \longrightarrow \mathcal{O}_{X_{p}}(1) \longrightarrow 0,
$$

where the second map is given by $\left(f_{1}, f_{2}, f_{3}\right) \rightarrow x f_{1}+y f_{2}+z f_{3}$.

By the above Corollary, we have

(1) if $p \equiv \pm-3(8)$ and $p \gg 0$ then $l=4$ and $s=1$, i.e. $V_{X_{p}}$ is semistable, and $F^{*}\left(V_{X_{p}}\right)$ is not semistable and has strongly semistable HN filtration and

$$
a_{1}\left(V_{X_{p}}\right)=\mu\left(V_{X_{p}}\right)+\frac{2}{p} \text { and } a_{2}\left(V_{X_{p}}\right)=\mu\left(V_{X_{p}}\right)-\frac{2}{p}
$$

In particular $\mu_{H K}\left(V_{X_{p}}\right)=2 \mu\left(V_{X_{p}}\right)^{2}+\frac{8}{p^{2}}$.

(2) if $p \equiv \pm-1(8)$ then $l=0$, i.e. $V_{X_{p}}$ is strongly semistable, and

$$
a_{1}\left(V_{X_{p}}\right)=\mu\left(V_{X_{p}}\right)
$$

In particular $\mu_{H K}\left(V_{X_{p}}\right)=2 \mu\left(V_{X_{p}}\right)^{2}$.

In particular, for $p \gg 0$ the numbers $a_{1}\left(V_{X_{p}}\right) a_{2}\left(V_{X_{p}}\right)$ do not eventually become constant or a well defined function of $p$, but keep oscillating and converge to $\mu\left(V_{X}\right)$. 


\section{REFERENCES}

[B1] H. Brenner, The rationality of the Hilbert-Kunz multiplicity in graded dimension two, preprint, arXiv:math.AC/0402180v1.

[B2] H. Brenner, A characteristic zero Hilbert-Kunz criterion for solid closure in dimension two, Math. Res. Lett., 11 (2004), no. 5-6, 563-574.

[BCP] R. Buchweitz, Q. Chen and K. Pardue, Hilbert-Kunz functions, preprint (Algebraic Geometry e-print series).

[C] Q. Chen, Hilbert-Kunz functions: a survey, unpublished notes May 1997, EssenSeminar.

[H] C. Han, The Hilbert-Kunz function of a diagonal hypersurface, Ph.D. thesis, Brandeis University (1991).

[HM] C. Han and P. Monsky, Some surprising Hilbert-Kunz functions, Math. Z., 214 (1993), no. 1, 119-135.

[HN] G. Harder and M. S. Narasimhan, On the cohomology groups of moduli spaces of vector bundles on curves, Math. Ann., 212 (1975), 215-248.

[L] A. Langer, Semistable sheaves in positive characteristic, Ann. Math., 159 (2004).

[Ma] M. Maruyama, Openness of a family of torsion free sheaves, J. Math. Kyoto Univ., 16-3 (1976), 627-637.

[M] P. Monsky, The Hilbert-Kunz function, Math. Ann., 263 (1983), 43-49.

[SB] N. I. Shepherd-Barron, Semistability and reduction mod p, Topology, 37 (1998), no. 3, 659-664.

[T1] V. Trivedi, Semistabiltiy and HK multiplicities for curves, J. of algebra, 284 (2005), 627-644.

[T2] V. Trivedi, Strong semistability and Hilbert-Kunz multiplicity for singular plane curves, to appear in Contemporary Mathematics of AMS.

[WY] K. Watanabe and K. Yoshida, Hilbert-Kunz multiplicity and an inequality between multiplicity and colength, J. Algebra, 230 (2000), 295-317.

School of Mathematics

Tata Institute of Fundamental Research

Homi Bhabha Road

Mumbai-400005

India

vija@math.tifr.res.in 